\title{
Desarrollo de Indicadores Bioquímicos de Calidad de Suelos del Sudoeste Bonaerense con Enmiendas de Residuos y Efluentes Agroindustriales
}

\section{Quality Bio-indicators development - Buenos Aires southwest soil with agroindustrial Wastes amendments}

Presentación: 6-7/10/2020

\section{Doctorando:}

\section{Victoria Alejandra Monserrat}

Grupo de Estudio Ambiente, Química y Biología, Facultad Regional Bahía Blanca, Universidad Tecnológica Nacional - Argentina victoriamonserrat@frbb.utn.edu.ar

\section{Director:}

\section{Horacio Campaña}

\section{Resumen}

El presente trabajo tiene como finalidad resumir el avance de la tesis doctoral "Desarrollo de Indicadores Bioquímicos de Calidad de Suelos del Sudoeste Bonaerense con enmiendas de Residuos y Efluentes Agroindustriales", la misma se lleva a cabo dentro del Grupo de Investigación GEAQB (Grupo de Estudio Ambiente, Química y Biología) en la Universidad Tecnológica Nacional Facultad Regional Bahía Blanca. El objetivo de la tesis es desarrollar indicadores de calidad ambiental para suelos del sudoeste bonaerense con enmienda de residuos orgánicos provenientes de la producción avícola intensiva. Para ello se propuso comparar los indicadores biológicos en suelo con enmienda orgánica, con y sin tratamiento, en contraste con suelo natural de forma tal de poder obtener correlaciones que vinculen distintos indicadores, como metodología de seguimiento y control de la calidad del suelo, incluyendo actividades enzimáticas, así como las relaciones entre dichas actividades y los parámetros de control físico químicos típicos. Se muestran los resultados parciales de caracterización de suelos, enmiendas, técnicas de determinación de parámetros bioquímicos y mediciones preliminares.

Palabras clave: Enzimas, Enmiendas Orgánicas, Indicadores de calidad de suelos.

\begin{abstract}
The purpose of this work is to summarize the progress of the doctoral thesis " Quality Bio-indicators development - Buenos Aires southwest soil with agroindustrial Wastesamendments, it is carried out within the GEAQB Research Group (Group of Study Environment, Chemistry and Biology) at the National Technological University Bahía Blanca Regional Faculty. The objective of the thesis is to develop environmental quality indicators for soils of the southwest of Buenos Aires with amendment of organic residues from intensive poultry production. It was proposed to compare the biological indicators in soil with organic amendment, with and without treatment, in contrast to natural soil in a way that was to be able to obtain correlations between different indicators, such as a methodology for monitoring and control of soil quality, including enzymes activities, as well as the relationships between these activities and typical physical-chemical control parameters. The partial results of soil
\end{abstract}


characterization, amendments, techniques for determining biochemical parameters determination and preliminary measurements are shown in this work.

Keywords: Enzymes, organic amendment, soil quality indicators

\section{Introducción}

En las últimas décadas las grandes cantidades de efluentes y excrementos provenientes de animales, generados por los sectores de producción intensiva, representan un potencial riesgo de contaminación e impacto negativo en el medio ambiente si no son tratados adecuadamente. Cuando los efluentes y estiércoles son desechados sin tratamiento o tratados de manera incorrecta, son una fuente de contaminación para el aire, el agua y el suelo. Además de ser una fuente de contaminación microbiológica para el hombre en particular y para la biota en general. Entre estos residuos se encuentran los guanos del sector avícola. Una gallina produce 100 gramos de guano por día, lo que al año representan unos 36,5 kilogramos de desechos que se pueden transformar en fertilizantes orgánicos, dándole valor agregado, y contribuyendo a la aplicación de los conceptos de una “economía circular” (Geissdoerfer M. et al, 2017) La gallinaza y las camas de aves de corral contienen materia orgánica que puede convertirse en bioenergía gracias a determinadas tecnologías de proceso. Uno de los métodos más comunes para el manejo de los excrementos avícolas es la digestión anaerobia, que produce biogás, una mezcla de gases con diferentes concentraciones de metano combustible y un digestato estabilizado que puede utilizarse como enmienda orgánica.

La enmienda de suelos con desechos orgánicos es uno de los métodos de disposición final más económicos, aunque deben ser tratados previamente para evitar la contaminación del medio. Para estos casos, la digestión anaeróbica aplicada a los residuos orgánicos es una opción adecuada de gestión y valorización económica de la cual puede obtenerse biogás y biosólidos del residuo estabilizado. El biogás puede utilizarse para la generación de energía y los biosólidos se pueden aprovechar como abonos orgánicos y mejoradores de suelos. La composición química del producto obtenido luego de la estabilización anaeróbica varía en función del tipo de materia prima usada y del tiempo de estabilización, en general se obtiene un producto orgánico estabilizado, de adecuada calidad sanitaria, con buena actividad biológica y un contenido de fermentos nitrosos y nítricos, lo que permite un buen complemento cuando se incorporan estos materiales a suelos de baja calidad (áreas marginalmente productivas).

La calidad del suelo puede definirse como la capacidad funcional del mismo, dentro de los ecosistemas vecinos, de modo que mantenga la productividad biológica, la calidad ambiental y promueva la salud de las plantas y animales. Por lo tanto, no existe un único indicador para determinar la calidad del suelo, debido a la variabilidad de sus propiedades (Doran, 2000). El conjunto de indicadores debería mostrar sensibilidad ante cambios que ocurran en un período de tiempo corto. Las mediciones que conformarían un grupo de indicadores, incluyen propiedades físicas, químicas y biológicas del suelo. Las actividades enzimáticas, destacan por su relación con el medio tanto biótico como abiótico, con un rol importante en la ecología microbiológica (Burns et al, 2013). Las enzimas del suelo se clasifican en dos grupos: extracelulares (exoenzimas) e intracelulares (endoenzimas).

Una variable de calidad de suelo, especialmente para la producción agrícola, es el contenido y calidad de la materia orgánica, presentando el inconveniente de que ésta cambia lentamente. Son necesarios muchos años para que se produzcan modificaciones medibles en un suelo debidos a cambios en el manejo del mismo (laboreo, rotación de cultivos, etc.). La determinación de la actividad biológica medida por la presencia de diferentes exoenzimas en el suelo, es un buen indicador de la participación de los microorganismos en los procesos de estabilización de la materia orgánica (Burns et al, 2013).Actualmente se ha acumulado evidencia de que las propiedades biológicas de un suelo son indicadores tempranos de estrés, haciéndolas 
idóneas para su uso en los diferentes programas de monitoreo. Los cambios en este tipo de parámetros proveen una identificación temprana de cambios en el estado y calidad del suelo.

Las enzimas intervienen (catalizan) importantes funciones del suelo, tales como el ciclo de nutrientes y descomposición de la materia orgánica y han sido utilizadas en la evaluación biológica de la calidad del suelo. Las enzimas más empleadas a tal fin están relacionadas con el ciclo del C, N y P, tales como $\beta$-glucosidasa, ureasa y fosfatasa (Gil-Sotres et al., 2005). Dentro de las enzimas más estudiadas se encuentran las oxidoreductasas, entre ellas las deshidrogenasas, catalasas y peroxidasas y las hidrolasas (sobre todo fosfatasas, proteasas y ureasas). Un menor número de estudios se ha realizado en los enzimas del tipo transferasas o liasas. La variación en la actividad enzimática está directamente asociada con la dinámica y la concentración poblacional de microorganismos en la capa superior del suelo. La incorporación de biosólidos a los suelos aumenta la población de microorganismos en ellos. Las enmiendas orgánicas incrementan la actividad de las enzimas en el suelo, dado que juegan un papel fundamental en los procesos de degradación y evolución de la materia orgánica, interviniendo en la mineralización y humificación de la misma (Negassa W. et al, 2011).

En general, la actividad enzimática depende de las características del suelo y su composición. En suelos con poca cantidad de materia orgánica la actividad enzimática depende más de enzimas intracelulares que serán más sensibles a factores medioambientales que las extracelulares. (Caravaca et al., 2002). Las deshidrogenasas actúan en la degradación de la materia orgánica y son indicadores importantes del metabolismo oxidativo y de la actividad microbiana que ocurre en los suelos. Por esta razón, suelen ser seleccionadas para evaluar en condiciones controladas, el efecto de bioabonos sobre la calidad de los suelos, estimada en términos del metabolismo oxidativo y su relación con los cambios que se generan en algunas propiedades físicas. La actividad de una sola enzima no puede ser indicativa de la calidad del suelo debido a la especificidad de cada una.

De las enzimas involucradas en el ciclo del C, la $\beta$-glucosidasaes la más utilizada en la evaluación de la calidad de suelos sujetos a diferentes procedimientos de manejo (prácticas culturales). Esta enzima completa el proceso hidrolítico convirtiendo los fragmentos de celobiosa a glucosa, removiendo glucosa desde los extremos no reductores de pequeños oligosacáridos. La ureasa es una hidrolasa relacionada con la transformación del nitrógeno orgánico a amoníaco, está involucrada en el ciclo del nitrógeno. Su presencia en el suelo es de origen microbiano, encontrándose en células vivas y en células que ya han sido degradadas, en forma libre asociada al humus o a los coloides del suelo. Algunos autores consideran que su correlación con la biomasa no es significativa y que es afectada por diversos factores como concentración de oxígeno, metales pesados y la disponibilidad de nitrógeno (Nayak D.R. et al, 2007). La mineralización de fósforo orgánico es llevada a cabo por un grupo de enzimas conocidas como fosfatasas o fosfohidrolasas que catalizan la hidrólisis de ésteres y de anhídridos de ácido fosfórico. Las mismas son enzimas extracelulares que son liberadas al suelo por microorganismos. Celulasas es el nombre genérico que recibe un complejo de enzimas capaces de degradar celulosa, un polisacárido formado por moléculas de glucosa unidas entre sí por enlaces $\beta-1,4$. El complejo enzimático está conformado por endo y exo-glucanasas, las cuales hidrolizan los enlaces $\beta-1,4$ de la celulosa para producir gluco-oligosacáridos y celobiosa, a partir de celulosa respectivamente (Bayer E.A. et al, 2004). La actividad de la deshidrogenasa, debido a que es parte integral de los microorganismos, se puede utilizar como un indicador de la actividad microbiana del suelo. Se localiza principalmente en las membranas plasmáticas de las bacterias o en las membranas mitocondriales de hongos y refleja la actividad oxidativa total de la biomasa microbiana (Giusquiani P. et al, 1995). La actividad proteasa es la responsable de la descomposición progresiva del nitrógeno que contienen las proteínas hasta obtener el nitrógeno de aminoácidos, cuyo origen se encuentra en la mayoría de los microorganismos contenidos en él y en residuos vegetales y de fauna.

El análisis de las actividades enzimáticas del suelo es una técnica relativamente sencilla y económica, a pesar de ello, la variabilidad de los resultados depende de diversos factores naturales y antropogénicos, entre los cuales se encuentran la localización geográfica, cambios estacionales, propiedades físico-químicas y contenido de materia orgánica, biomasa microbiana, contenido de arcillas, aplicación de agroquímicos, contaminación y el manejo del suelo. Diversos autores proponen integrar las actividades enzimáticas en un índice que cumpla como mínimo las siguientes condiciones: i) reflejar los cambios de 
calidad de suelo debido a distintos factores, ii) mostrar baja variabilidad estacional, iii) el número de variables elegidas para el índice debe ser el menor posible y su medida debe ser simple y rápida y iv) las variables elegidas deben estar relacionadas con procesos bioquímicos o biológicos del suelo.

\section{Desarrollo}

\section{Materiales y Métodos}

El suelo utilizado corresponde a un establecimiento agrícola ganadero ubicado en la localidad de Cabildo, partido de Bahía Blanca ( $38^{\circ} 30^{\prime} \mathrm{S}, 62^{\circ} 50^{\prime} \mathrm{O}, 152$ m.s.n.m.) en el sudoeste bonaerense. El terreno de las experiencias presenta un relieve suavemente ondulado o casi horizontal y corresponde al tipo de suelo Argiustol típico (Campaña et al. 2016). Las muestras de suelo fueron secadas al aire, homogeneizadas y tamizadas con malla de $2 \mathrm{~mm}$.

Para las determinaciones físico-químicas se utilizaron los Métodos Normalizados del Standard Methods (SMWW, 1999).Los resultados fueron los siguientes: $\mathrm{CE}=0,3 \mathrm{mS} . \mathrm{cm}^{-1} ; \mathrm{MO}=29,2 \mathrm{~g} \mathrm{~kg}^{-1} ; \mathrm{C}=16,9 \mathrm{~g} \mathrm{~kg}^{-1} ; \mathrm{N}=1,1 \mathrm{~g} \mathrm{~kg}^{-1} ; \mathrm{NO}_{3}+\mathrm{NO}_{2}=60 \mathrm{mg} \mathrm{kg}{ }^{-1}$, $\mathrm{NH}_{4}{ }^{+}=20 \mathrm{mg} \mathrm{kg}{ }^{-1} ; \mathrm{C} / \mathrm{N}=16 ; \rho$ real $=2,56 \mathrm{Mg} \cdot \mathrm{m}^{-3} ; \rho$ aparente $=1,33 \mathrm{Mg} \cdot \mathrm{m}^{-3} ;$ arena $=60 \% ;$ limo $=20 \%$; arcilla $=20 \%$; capacidad de retención de agua $35 \%$.

Los ensayos de determinación de actividad enzimática se realizaron por triplicado, siguiendo los métodos descriptos en "Methods in AppliedSoilMicrobiology and Biochemistry", (Alef et al. 1995). La estimación de la fosfatasa ácida se basó en la determinación del p-nitrofenol liberado por la actividad de la enzima cuando el suelo fue incubado con una solución de pnitrofenil fosfato disódico $(\mathrm{pH}$ 6,5).La actividad de la proteasa se estimó mediante la determinación de los aminoácidos liberados luego de la incubación de la muestra en presencia de caseinato de sodio. En el caso de la $\beta$-glucosidasa, debido a que hidroliza el p-nitroß-D-glucopiranósido liberando p-nitrofenol se utilizó el método propuesto por Tabatabai,1982 / Eivazi y Tabatabai, 1988.La determinación de las celulasas totales se basa en la determinación de los azúcares reductores producidos luego de la incubación de la muestra en presencia de celulosa microcristalina (Avicel) como sustrato. La ureasa, cataliza la hidrólisis de la urea, produciendo $\mathrm{CO}_{2}$ y $\mathrm{NH}_{3}$.Por tanto su actividad se estimó mediante la determinación del $\mathrm{NH}_{4}{ }^{+}$liberado a partir del suelo incubado con una solución de urea. En la medición de la absorbancia para la determinación de las actividades enzimáticas se utilizó un espectrofotómetro HACH DR 2010.

La biomasa residual utilizada es guano de gallina de una granja de producción intensiva situada sobre la $\mathrm{RN} \mathrm{N}^{\circ} 51$ en cercanía de la zona de Bahía Blanca, la caracterización se realizó para guano fresco y seco mezclado con suelo. Para la determinación físico química de $\mathrm{pH}, \mathrm{MS}, \mathrm{MO}, \mathrm{CE}$, CO, Sólidos Volátiles, NTK SS y $\mathrm{PO}_{4}^{-3}$ se utilizaron los Métodos Normalizados 2540 B, 2540 C, 2540 D, 2540 E, 2540 G y 5220 D del Standard Methods (SMWW, 1999).

\section{Diseño Experimental:}

Los ensayos con biosólidos residuales en suelo se llevarán a cabo en macetas, (laboratorio), de forma tal de independizar el suelo y las enmiendas estudiadas de las condiciones ambientales (temperatura, humedad, etc.) y lograr un ambiente controlado. Los mismos se realizarán en macetas por triplicado, con 3 dosis de estiércol de gallina con y sin estabilización, comparando con suelo de control sin aplicación de enmienda, totalizando una cantidad de 21 macetas. Estos ensayos se realizarán en condiciones de temperatura controlada a $25^{\circ} \mathrm{C}$ y humedad de suelo equivalente al $50 \%$ de la capacidad de campo. Se utilizarán macetas de PEAD (Polietileno de alta densidad) y se colocará aproximadamente $1 \mathrm{~kg}$ de suelo por maceta. La enmienda a utilizar se aplicará en dosis de lodo equivalentes considerado el aporte de nitrógeno del biosólido y su tasa de mineralización que puede ser de aproximadamente el $40 \%$ durante el período de ensayo. La cantidad a aplicar por maceta se calcula en función del Nitrógeno del digestato, considerando 3 dosis: baja, media y alta $(100-250-500 \mathrm{~kg} \mathrm{~N} / \mathrm{ha})$. La duración del ensayo será de 120 días y las mismas serán muestreadas a los 0,60 y 120 días. 


\section{Resultados preliminares}

Actividad enzimática del suelo natural: las enzimas hidrolíticas analizadas y los resultados de actividades medias fueron los siguientes:

- $\quad \beta$-glucosidasas: $0.126 \mu \mathrm{mol} \mathrm{p}$-Nitrophenol $\mathrm{g}^{-1} \mathrm{~h}^{-1}$.

- Ureasas: $0.6882 \mu \mathrm{mol} \mathrm{N}-\mathrm{NH} 4+\mathrm{g}^{-1} 2 \mathrm{~h}^{-1}$

- Proteasas: $0.019 \mu \mathrm{mol}$ tirosina $\mathrm{g}^{-1} 2 \mathrm{~h}^{-1}$

- Celulasas: $0.287 \mu \mathrm{mol}$ de glucosa $\mathrm{g}^{-1} 16 \mathrm{~h}^{-1}$

- Fosfatasas: $0.5522 \mu \mathrm{mol} \mathrm{PNP} / \mathrm{g}^{-1} \mathrm{~h}^{-1}$

Los valores obtenidos de $\beta$-glucosidasas son de igual orden a los publicados por Celis (2011) quién obtuvo actividades enzimáticas de entre 0.3 y $0.6 \mu \mathrm{mol}$ p-Nitrophenol g-1 h-1 para suelos de control sin enmendar y sin cultivar. Para suelos degradados Bastida (2008) publicó actividades de $2.3 \mu \mathrm{mol}$ p-Nitrophenol g ${ }^{-1} \mathrm{~h}^{-1}$.

En el caso de las ureasas, los valores obtenidos son similares a los hallados en suelos por Negassa (2011), quien obtuvo una actividad de $0.56 \mu$ mol N-NH4 $+\mathrm{g}^{-1} 2 \mathrm{~h}^{-1}$ con $2.4 \mathrm{~g} \mathrm{~kg}^{-1}$ de $\mathrm{N}$ total, mientras que Celis (2011) refiere valores de entre 1 y 1.2 $\mu$ mol N-NH4 $+\mathrm{g}^{-1} 2 \mathrm{~h}^{-1}$ para el caso de suelos sin enmendar. El mismo autor ha publicado valores de entre 0.7 y $4.5 \mu \mathrm{mol}$ PNP/ $\mathrm{g}^{-1} \mathrm{~h}^{-1}$ para la actividad de fosfatasas en el mismo tipo de suelo, mientras que para suelos agrícolas de la Región Pampeana se han obtenido actividades de hasta $41.15 \mu \mathrm{mol}$ PNP/ g-1 h-1, Fernández et. al. (2008). La diferencia en este caso podría deberse a la presencia de un suelo con mayor cantidad de fósforo que el del suelo bajo estudio.

La bibliografía refiere para la actividad de las proteasa valores de entre 4.96 y $0.55 \mu$ mol tirosina $\mathrm{g}^{-1} \mathrm{~h}^{-1}$ para ensayos realizados en suelos bajo diversas especies forestales y en distintas estaciones del año, Defrieri (2008). Los valores corresponden a sitios con mayor cantidad de nutrientes por lo que la actividad resulta mayor debido a que las proteasas catalizan las reacciones que degradan proteínas y péptidos liberando aminoácidos.

Bautista Cruz (2014) ha publicado estudios en suelos arcillosos con pH 8.20, $1.37 \%$ COT y $0.11 \%$ NTK donde las actividades de celulasas han arrojado valores de 2.2x10-4 $\mu$ mol de glucosa $\mathrm{g}^{-1} 24 \mathrm{~h}^{-1}$, mientras que Guiwei (2008) obtuvo valores $0.012 \mu$ mol de glucosa g-1 16h-1 en suelos con pH ácidos y baja cantidad nutrientes, que los resultados obtenidos sean superiores los publicados deberse a mayor presencia de nutrientes vegetales.

En la Tabla 1 se muestran los resultados de la caracterización físico-química de la biomasa residual sin estabilizar, donde GF corresponde a los resultados para Guano de Gallina Fresco y GS para Guano de Gallina Seco mezclado con suelo

\begin{tabular}{|c|c|c|c|c|c|c|c|c|}
\hline & $\mathbf{p H}$ & $\begin{array}{c}\mathbf{C E} \\
\mathbf{m S} / \mathbf{c m}\end{array}$ & $\mathbf{M S} \%$ & $\mathbf{M O} \%$ & $\mathbf{C O} \%$ & $\mathbf{S V} \%$ & $\begin{array}{l}\mathbf{N T K}-\mathbf{S S} \\
\mathbf{\%}\end{array}$ & $\begin{array}{l}\mathbf{P O}_{\mathbf{4}}{ }^{-3} \\
\mathbf{m g} / \mathbf{L}\end{array}$ \\
\hline $\mathbf{G F}$ & 6,65 & 8,90 & 21,43 & 64,37 & 37,34 & 68,91 & 3,13 & 128,5 \\
\hline GS & 8,27 & 4,84 & 70,57 & 31,89 & 18,53 & 38,09 & 1,27 & 160 \\
\hline
\end{tabular}

Tabla 1: caracterización físico química de biomasa residual

\section{Referencias}

Alef, K; Nannipieri, P. (1995). Methods in Applied Soil Microbiology and Biochemistry. ISBN: 978-0-12-513840-6.

Bastida, F; Kandeler, E; Moreno, J. L.; Ros, M; García, C; Hernández, T. Application of fresh and composted organic wastes modifies structure, size and activity of soil microbial community under semiarid climate. Applied soil ecology 40 (2008) 318329. 
Bautista-Cruz, A; Cruz Domínguez, G; Rodríguez Mendoza, M; Pérez Pacheco, R; Robles, C. Effect of compost and slowrelease fertilizers addition on soil biochemistry and yield of maize (Zea mays L.) in Oaxaca, Mexico. Rev. FCA UNCUYO. 2014. 46(1): 181-193. ISSN impreso 0370-4661. ISSN (en línea) 1853-8665.

Bayer E, Belaich J, Shoham Y, Lamed R, The Cellulosomes: Multienzyme Machines for Degradation of Plant Cell Wall Polysaccharides, Annu. Rev. Microbiol (2004). 58:521-54

Burns, R. G; De Forest, J. L. Marxsen, J; Sinsabaugh, R. L; Stromberger, M. E; Wallenstein, M. W; Weintraub, M. N; Zoppini, A. 2013. Soil enzymes in a changing environment: Current knowledge and future directions. Soil Biology and Biochemistry. Vol. 58, pp 216-234.

Campaña, D H, Airasca A O., Andrade Couce M L. Limiting factors for pasture and cereal production in marginal soils of the southwestern Pampas in the province of Buenos Aires, Argentina. International Journal of Environmental \& Agriculture Research (IJOEAR) (2016). ISSN:2454-1850 vol-2, issue-12.

Caravaca, F; Masciandaro, G; Ceccanti, B. 2002. Land use in relation to soil chemical and biochemical properties in a semiarid Mediterranean environment. Soil \& Tillage Research, 68, 23-30.

Celis J, Machuca A, Sandoval M, Morales P. Biological Activity in a degraded Alfisol amended with sewage sludge and cropped with yellow serradela (Ornithopuscompressus L.)Chilean Journal of AgriculturalResearch (2011) 71(1):164-172

Defrieri, R.L; Effron, D; Jimenez, M.P; Prause, J.2008. Influencia de especies forestales sobre la actividad de lasenzimas fosfatasa ácida y proteasas en un suelo de bosque. CI. Suelo (Argentina) 26(2): 177-182.

Doran, John W. y Zeiss, Michael R. Soilhealth and sustainability: managing the biotic component of soil quality. Applied Soil Ecology 15 (2000) 3-11.

Fernández L, Sagardoy M. Gómez M. Estudio de la fosfatasa ácida y alcalina en suelos de la región Pampeana Norte del área sojera. CI. Suelo Argentina (2008) 26(1): 35-40.

Geissdoerfer, M; Savaget, P; Bocken N. M. P; Hultink, E. J. 2017. The Circular Economy e A new sustainability paradigm? Journal of Cleaner Production 143, 757-768.

Gil - Sotres, F; Trasar - Cepeda, C; Leirós, M. C; Seoane, S. 2005. Different approaches to evaluating soil quality using biochemical properties. Soil Biol. Biochem., 37: 877-887.

Giusquiani P, Pagliai M, Gigliotti G, Businelli D, Benetti A. Urban Waste Compost: Effects on physical, chemical and biochemical soil properties. J. Environ. (1995) Qual. 24: 175 - 182

Guiwei, Q., de Varennes, A., \& Cunha-Queda, C. (2008).Remediation of a mine soil with insoluble polyacrylate polymers enhances soil quality and plant growth. Soil Use and Management, 24, 350-356.

Nayak D, Babu Y, Adhya T. Long-term application of compost influences microbial biomass and enzyme activities in a tropical Aeric Endoaquept planted to rice under flooded condition. Soil Biol. Biochem. (2007) 39: 1897-1906.

Negassa W, Baum C, Leinweber, L. Soil amendment with agro-industrial byproducts: molecular-chemical compositions and effects on soil biochemical activities and phosphorus fractions. Journal of plant nutrition and soil science. (2011) 174, 113-120.

SMWW (1999).Standard Methods for the examination of Water and Wastewater.20th edition. American Public Health Association. American Water Works Association. Water Environment Federation. 